\title{
EMMA - the Electric and Magnetic Monitor of the Aurora on Astrid-2
}

\author{
L. G. Blomberg ${ }^{1}$, G. T. Marklund ${ }^{1}$, P.-A. Lindqvist ${ }^{1}$, F. Primdahl ${ }^{2,3}$, P. Brauer ${ }^{2}$, L. Bylander ${ }^{1}$, J. A. Cumnock ${ }^{1,4}$,

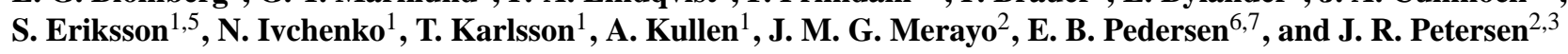 \\ ${ }^{1}$ Alfvén Laboratory, Royal Institute of Technology, Stockholm, Sweden \\ ${ }^{2}$ Ørsted DTU, Technical University of Denmark, Lyngby, Denmark \\ ${ }^{3}$ Danish Space Research Institute, Copenhagen, Denmark \\ ${ }^{4}$ Also at University of Texas at Dallas, Richardson, TX, USA \\ ${ }^{5}$ Now at University of Colorado, Boulder, CO, USA \\ ${ }^{6}$ TERMA Electronics AS, Lystrup, Denmark \\ ${ }^{7}$ Now at Ericsson Telebit A/S, Viby J., Denmark
}

Received: 30 December 2002 - Revised: 26 April 2003 - Accepted: 28 May 2003 - Published: 1 January 2004

\begin{abstract}
The Astrid-2 mission has dual primary objectives. First, it is an orbiting instrument platform for studying auroral electrodynamics. Second, it is a technology demonstration of the feasibility of using micro-satellites for innovative space plasma physics research. The EMMA instrument, which we discuss in the present paper, is designed to provide simultaneous sampling of two electric and three magnetic field components up to about $1 \mathrm{kHz}$. The spin plane components of the electric field are measured by two pairs of opposing probes extended by wire booms with a separation distance of $6.7 \mathrm{~m}$. The probes have titanium nitride (TiN) surfaces, which has proved to be a material with excellent properties for providing good electrical contact between probe and plasma. The wire booms are of a new design in which the booms in the stowed position are wound around the exterior of the spacecraft body. The boom system was flown for the first time on this mission and worked flawlessly. The magnetic field is measured by a tri-axial fluxgate sensor located at the tip of a rigid, hinged boom extended along the spacecraft spin axis and facing away from the Sun. The new advanced-design fluxgate magnetometer uses digital signal processors for detection and feedback, thereby reducing the analogue circuitry to a minimum. The instrument characteristics as well as a brief review of the science accomplished and planned are presented.
\end{abstract}

Key words. Ionosphere (auroral ionosphere). Magnetospheric physics (magnetosphere-ionosphere interactions). Space plasma physics (instruments and techniques)

\section{Introduction}

Astrid-2 is a Swedish micro-satellite launched on 10 December 1998 into an $83^{\circ}$ inclination circular orbit at $1000 \mathrm{~km}$

Correspondence to: L. G. Blomberg

(Lars.Blomberg@alfvenlab.kth.se) altitude. It remained operational until 24 July 1999 when the spacecraft ceased to telemetre data to the ground. The orbital plane regresses $360^{\circ}$ relative to the Sun in approximately 7 months, so during the lifetime of the spacecraft the orbit covered all local times. To facilitate the inclusion of adequate instrumentation on a space and mass constrained micro-satellite, significant new instrument development was necessary. Holback et al. (2001) describe the Langmuir probe instrument and Norberg et al. (2001) describe the particle detectors and UV imagers. Overviews of the Astrid-2 mission are given by Blomberg et al. (1999) and Marklund et al. (1997b; 2001a). Here we describe the electric and magnetic field instrument EMMA.

The primary quantities measured by EMMA are two components of the electric field and the full vector magnetic field. The electric sensors are of the biased double-probe type and the magnetic sensors use the flux-gate technique. In addition to measuring the electric field by current-biasing the electric probes, they may also be used to estimate plasma density and temperature by means of sweeping the bias to obtain a current-voltage characteristic. From this, information about the coupling between the probe and the plasma may be derived as well. Sampling is done at 16, 256, or 2048 samples per second. EMMA is equipped with $12 \mathrm{MB}$ semiconductor memory for storage of data from times without ground station contact and also for use with the highest sampling rate where the data throughput exceeds the capacity of the telemetry link.

The EMMA instrument worked very well during its seven months of operation. A wealth of scientific data was collected and significant progress on its interpretation has already been made, as evidenced by several already published papers. In the present paper the design and capabilities of the EMMA instrument are described. We also briefly review some results obtained as well as the potential for future research. 


\section{Scientific Objectives and Results}

The EMMA instrument, together with the other instruments on board Astrid-2, allows for a number of scientific topics to be addressed. Below is a list of examples of topics, studied or planned for future study.

- Sources of the Cross-Polar Potential Drop. The crosspolar potential drop arises from the solar wind's interaction with Earth's magnetic field. EMMA has provided new and valuable data that may deepen our understanding of the interaction mechanisms (e.g. Eriksson et al., 2001).

- Electric Fields and Currents in the Nightside Convection Throat. Enhanced electrojet activity is often associated with an auroral bulge in the nightside ionosphere. Astrid-2 data have demonstrated that current continuity across the bulge region is not necessarily maintained by polarisation electric fields, as in the Cowling channel model, but rather by local field-aligned currents flowing in both directions (Marklund et al., 2001b).

- Transpolar Aurorae. The high-inclination orbit of Astrid-2 puts it in an ideal position for studying transpolar auroral arcs. A thorough investigation, combining Astrid-2 data with Polar UVI and DMSP data is underway. Initial results on the configuration of plasma flows and field-aligned currents in transpolar arcs are presented by Blomberg and Cumnock (submitted manuscript, Adv. Space Res., 2002).

- Global Mapping of $\boldsymbol{E}, j_{\|}, \boldsymbol{B}$. To understand the fundamental relationship between the ionospheric electric field and the field-aligned currents on a global scale, statistical surveys based on data from the same platform are needed (e.g. Blomberg et al., 1992). Such pictures are normally not found in the literature. EMMA data were recently used to ascertain the Weimer $2 \mathrm{~K}$ ionospheric electric field model (Eriksson et al., 2002). EMMA data have also been used as a complement to the Danish Ørsted satellite for mapping of the geomagnetic field (Merayo et al., 2002).

- Waves and Pulsations up to $1 \mathrm{kHz}$. With a maximum sampling rate of the field instruments of $2048 \mathrm{~s}^{-1}$ wave frequencies up to the local proton gyrofrequency are covered. Thus, a multitude of pure wave as well as wave-particle interaction phenomena may be studied. Ivchenko and Marklund (2001) discuss initial results.

- Cusp Topology and Dynamics. Astrid-2 passes through the cusp regularly whenever the orbital plane has a noon-midnight orientation. Using the particle and field instruments on board will allow for detailed studies of the low-altitude signatures of the polar cusp. Initial results were presented by Keith et al. (2001).

- Ion Clouds and Ion Injection. Høymork et al. (2001) studied dense ion clouds in the inner magnetosphere and discussed their possible relation to substorm ion injection. Further study of the particle characteristics and the plasma flows associated with these events will shed light on the ion injection mechanisms.

- Spacecraft-Plasma Interaction. Any spacecraft disturbs the surrounding plasma to an extent that depends on the spacecraft design and how it is operated, as well as on the properties of the plasma it traverses. The spacecraft charges electrically, which creates an electrostatic barrier around it. Since the spacecraft moves at $7 \mathrm{~km} / \mathrm{s}$ it leaves a wake behind it. Understanding these effects more thoroughly is important for a complete understanding of the instrument's performance. Initial results on the interaction of Astrid-2 with the plasma environment based on EMMA and LINDA data were presented by Ivchenko et al. (2001).

- Electrodynamics of Aurora and Black Aurora. A discovery by the Freja spacecraft (Marklund et al., 1994; 1997a) illustrates the complexity of field-aligned acceleration processes. An anti-symmetry exists between the electric field structures in the upward field-aligned current region associated with auroral particles and those in the downward current region. Freja demonstrated the existence of upward acceleration of electrons in these regions, leading to the formation of ionospheric density cavities and associated strong transverse electric fields. The upward acceleration region is typically found at lower altitudes than the auroral acceleration regions, and EMMA can contribute significant new observational data to shed additional light on the processes operating.

- Fine-Structure of Electric Fields and Field-Aligned Currents. Sharp gradients in the electric and magnetic field data are frequently observed. These may be associated with localized very strong field-aligned currents. This phenomenon was studied by Ivchenko and Marklund (2002).

- Sub-Auroral and Equatorial Electric Field Structures. Strong poleward directed electric fields are commonly observed at sub-auroral latitudes in the pre-midnight sector. They are believed to be associated with closure of field-aligned currents through the mid-latitude trough, an ionospheric region of depleted plasma density and, thus, low conductivity. For an overview of observational results, see Karlsson et al. (1998). Recent work, based on Astrid-2, is discussed by Figueiredo (2001) and by Figueiredo et al. (submitted manuscript, Ann. Geophys., 2002).

- Physics of Transverse Ion Heating. Transverse ion heating is thought to be an important mechanism for ion outflow from the upper atmosphere. The heating often takes place at fairly low altitude, and so, Astrid- 2 is in a good position to measure in situ the fields, waves, and particle properties associated with the heating process. 
Table 1. Summary of measured quantities

\begin{tabular}{lllll}
\hline Quantity & Range & Resolution & Bits & Sample rate $\left(\mathrm{s}^{-1}\right)$ \\
\hline E (2 components) & $\pm 5 \mathrm{~V} / \mathrm{m}$ & $0.03 \mathrm{mV} / \mathrm{m}$ & 16 & $16,256,2048$ \\
B (3 components) & $\pm 62 \mu \mathrm{T}$ & $0.12 \mathrm{nT}$ & 20 & $16,256,2048$ \\
\hline
\end{tabular}

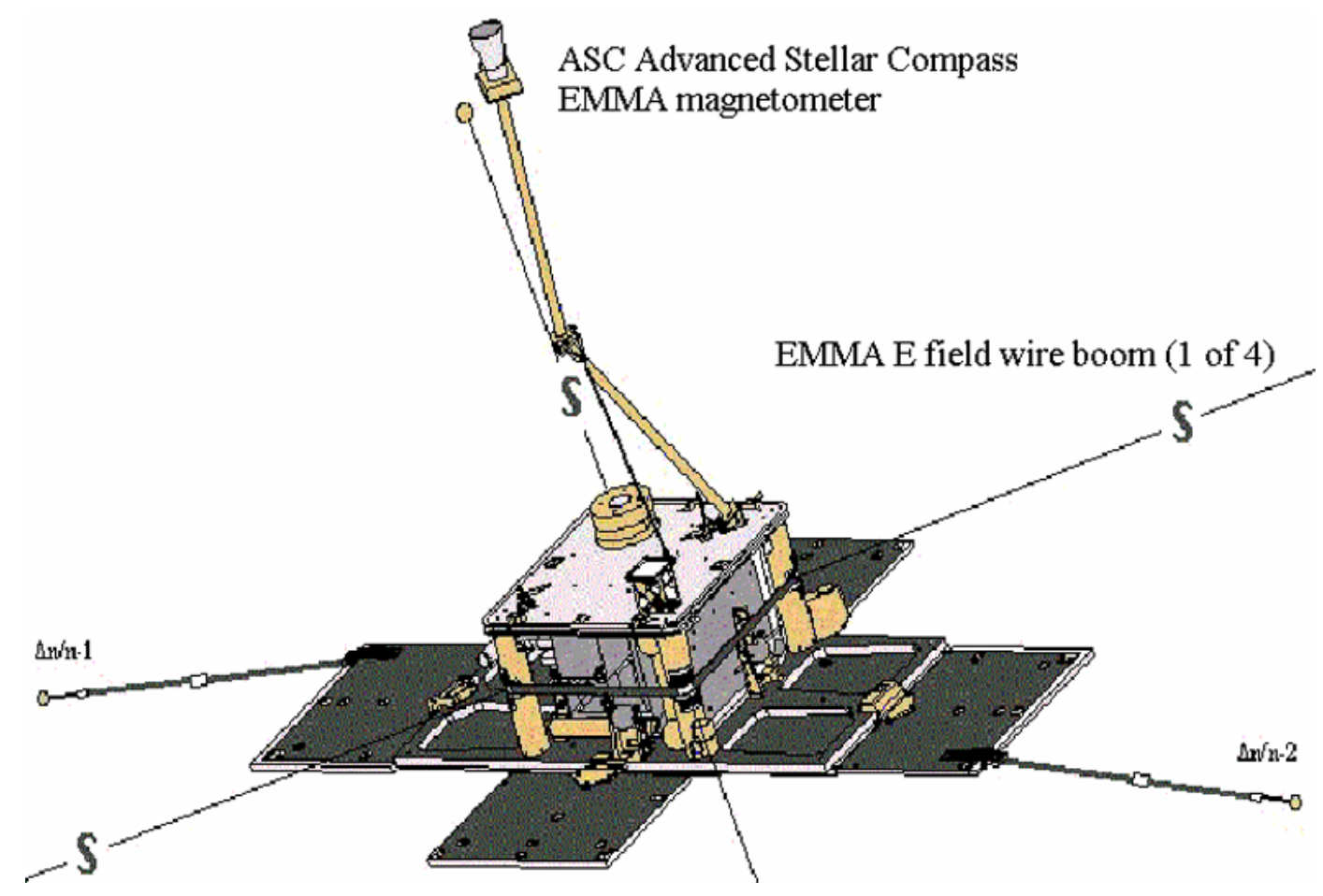

Fig. 1. EMMA sensor locations.

- $\boldsymbol{E}-\boldsymbol{B}$ Correlation and its Scale Size Dependence. Electric and magnetic fields associated with static structures in the ionosphere are often correlated. The electric field maps, at least partially, upwards along the geomagnetic field, and the field-aligned current produces a transverse magnetic disturbance field. Assuming complete mapping of the electric field the height-integrated ionospheric conductivity may be inferred from the ratio of the magnetic to the electric field. At least for large-scale sizes the electric field normally maps well between different altitudes. Studying the degree of correlation for smaller scale sizes may yield additional information about the ionosphere-magnetosphere interaction processes.

\section{Instrument design}

EMMA is designed to measure two components of the electric field and three components of the magnetic field simultaneously with high bit resolution and variable time resolution (see Table 1). The instrument box contains eight printed circuit boards, holding the electronics for the electric sensors, for the fluxgate sensors, for sampling and digitizing of all electric and magnetic sensor signals, for the boom motor control, as well as for the LINDA instrument (Holback et al., 2001). EMMA also has a 12 MB data memory for temporary storage of data either when outside of ground-station contact or when sampling at a data rate exceeding the capacity of the telemetry link.

Four spherical sensors located in the spin plane and extended away from the spacecraft using wire booms measure two components of the electric field (see Fig. 1). The deployment system for the wire booms is of a new design where the wire in the stowed configuration is wound around the perimeter of the spacecraft body. Each wire runs through a small loop, which is attached to a belt driven by a stepper motor. During boom deployment the stepper motor moves the belt up to $2 \mathrm{~mm}$ per second. As the belt moves the wires are unwound from the spacecraft body. In the deployed configuration the centre of each probe is located $3.35 \mathrm{~m}$ from the centre of the spacecraft. The deployment mechanism is illustrated in Fig. 2. 


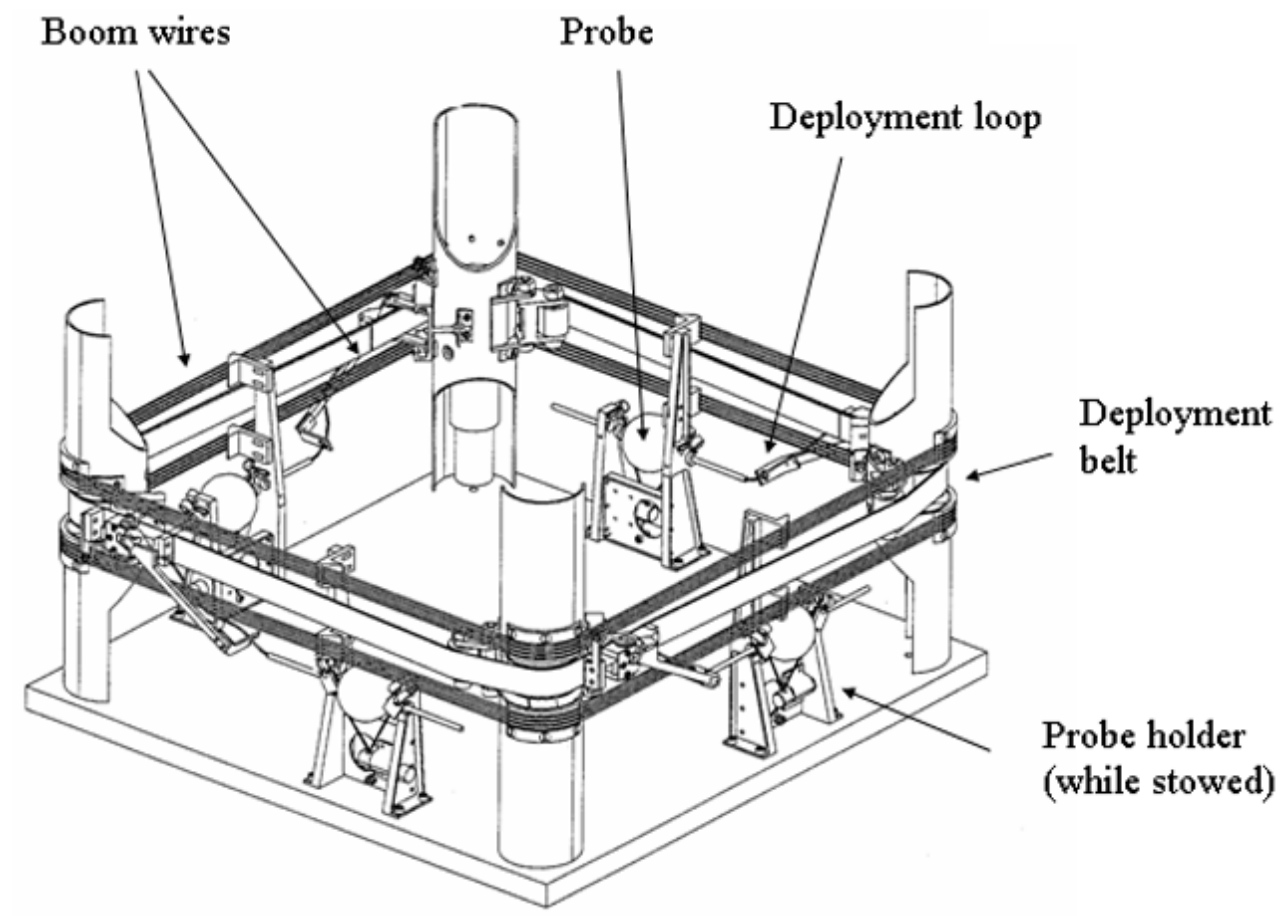

Fig. 2. EMMA wire boom mechanisms.

The total mass of the boom system including the deployment mechanisms is less than $2 \mathrm{~kg}$, compared to about $14 \mathrm{~kg}$ for four conventional mechanisms. For Astrid-2, the booms were fairly short, but there are no fundamental reasons why the new design could not allow for considerably longer boom lengths with only a slight increase in mass. In addition, there are variations of the design that may prove even more viable for longer booms. Thus, even though long and low-mass wire booms were not flight tested before the Astrid-2 launch, the successful in-flight performance proved that a significant reduction of the mass of future boom systems is attainable without compromising reliability. Figure 3 shows the boom system in the stowed position. A more detailed description of the low-mass wire boom system is found elsewhere (Hellman, 1996).

The sensors each have a mass of $100 \mathrm{~g}$, a diameter of $35 \mathrm{~mm}$, and a titanium nitride (TiN) surface. TiN has proved to have excellent surface properties, providing clean electrical contact between the probe and the surrounding plasma. This is evidenced, for example, by an almost complete lack of hysteresis in the current-voltage characteristic of the probes. TiN is used also for the LINDA probes on Astrid2, as well as on the Cassini mission (e.g. Kurth et al., 2001) and is likely, due to its superior properties compared to more traditional coatings such as DAG-213 or vitreous carbon, to become a new standard for probe surface material in space. Figure 4 shows one of the four EMMA probes.

Before sampling, the probe signals are amplified by preamplifiers located inside the spacecraft body. Ideally, preamplifiers should be mounted as close to the sensor as possible. Often the pre-amplifiers are mounted inside the spherical sensors, but with the relatively short boom length on Astrid2 this is not necessary, resulting in a reduction of complexity without loss of instrument performance. The signals also pass through low-pass filters designed to suppress aliasing from the sampled signals. The filtering is the same for all (electric and magnetic) sensor signals. The signals from the four electric field probes are sampled individually as voltages with respect to the satellite body, and are telemetred individually to the ground. This allows for computational reconstruction of the satellite potential with full time resolution, which helps with identifying rapid fluctuations in the plasma density, as well as time intervals, where one or more of the probes are saturated due to spacecraft charging. Each of the four probes can be fed with individually set bias currents, in order to optimize the quiescent point on the currentvoltage characteristic, or equivalently, to minimize the contact impedance between probe and plasma to make the measurement as insensitive as possible to variations in the ambient plasma density or temperature. Low contact impedance provides for as clean as possible a measurement of the probe potential with respect to the plasma. The bias current can be selected either in the range $\pm 40 \mathrm{nA}$ in $20 \mathrm{pA}$ increments or in the range $\pm 450 \mathrm{nA}$ in $220 \mathrm{pA}$ increments. In addition, the bias currents of one or more probes may be stepped through an adjustable sequence of values (known as a current sweep) at an adjustable rate, in order to register the current-voltage characteristics of the probe with respect to the plasma. This is useful for gaining information needed both for optimizing the choice of bias current and for determining plasma and probe properties, such as density, temperature and photoemission characteristics. 


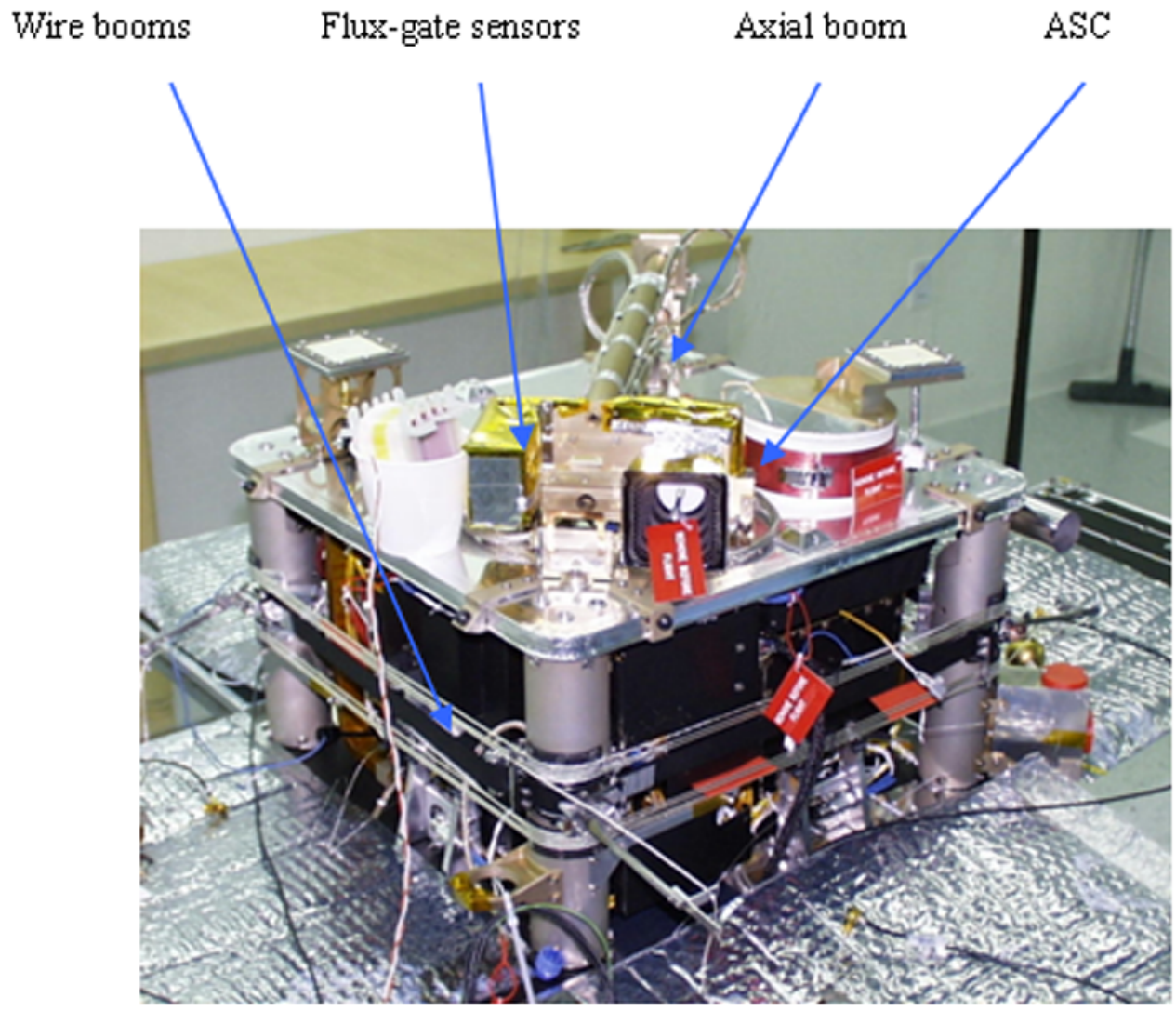

Fig. 3. The Astrid-2 platform.

The fluxgate sensors are located at the end of a $0.9 \mathrm{~m}$ long rigid boom extending axially from the platform on the side facing away from the Sun (see Fig. 3). The three sensors are nominally orthogonal to each other, and the sensor unit is co-located with the ASC (Advanced Stellar Compass) camera head, in order to optimize the attitude determination of the sensor axes (Jørgensen, 2000; Jørgensen et al., 2003). The fluxgate measurements are based on a principle first described by Piil-Henriksen et al. (1996), and the sensor design is developed from the one used in the Ørsted mission (Brauer et al., 2000). The raw fluxgate sensor signals are digitally sampled and then three DSPs (Digital Signal Processors) are used to perform the field extraction and the generation of the feedback currents for the sensors, driven by three digitalto-analogue converters (DAC). The excitation frequency is $8 \mathrm{kHz}$, and the excitation circuit uses parametric amplification to reduce power consumption. The algorithms executed by the DSPs form the core of the magnetometer. A more thorough description of the magnetometer is given by Pedersen et al. (1999). More technical details on EMMA are found in Blomberg et al. (2003).

The ASC was an add-on experiment in an attempt to obtain an early test flight of the Ørsted core instruments. It got a number of good star sky pictures, and it provided attitude information for limited periods. Because of the challenges involved in adapting the Ørsted (three-axis stabilized) ASC to the spinning Astrid-2 platform, an alternative method for attitude determination was developed in parallel at Ålborg University, Denmark (Bak, 1999), in part based on the Ørsted attitude control algorithms. The method uses magnetometer data only to build a dynamical model of the spacecraft spin and orientation in space. The method has proved to work well, routinely providing spacecraft attitudes at the $0.1^{\circ} \mathrm{ac}-$ curacy level, compared to the ASC attitudes acquired, as well as based on a statistical analysis. Magnetometer calibrations were performed on an instrument level at the "Magnetsrode" test facility of the Institute of Geophysics and Meteorology, Technical University of Braunschweig, Germany, in the laboratory at DTU and at the Danish Meteorological Institute's Magnetic Observatory at Brorfelde, Denmark (Brauer et al., 2000). The final satellite-level magnetic calibration was performed at the Swedish Geological Survey's Magnetic Observatory at Lovö near Stockholm, Sweden. The operating and near-flight configured satellite was exposed to the monitored Earth's field in about 60 different directions, and a scalar calibration was performed. Taking a local field gradient into account and compensating for the DAC's non-linearities, the overall standard deviation of the individual data points was $1.3 \mathrm{nT}_{\mathrm{rms}}$. This includes all the perturbations from the satellite and the remaining non-linearities in the sensor and the DACs. At the quasi-DC to $16 \mathrm{~Hz}$ filtered output and in a $\mu$-metal magnetic shield, the instrument's band noise was 


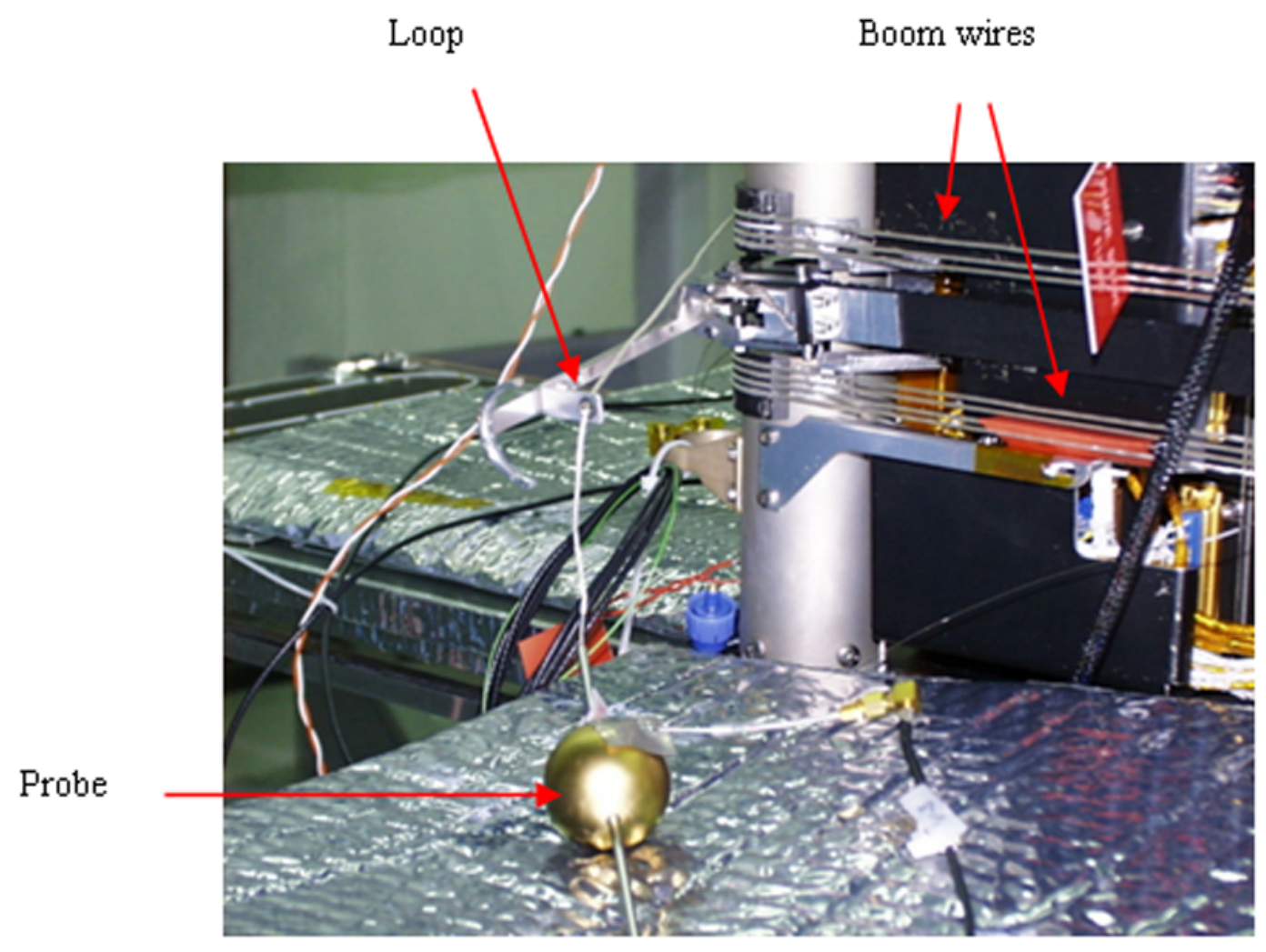

Fig. 4. The EMMA electric field probe and deployment mechanism.

below $50 \mathrm{pT}_{\text {rms }}$ (Pedersen et al., 1999). Tests at Lovö confirmed the very low magnetic perturbations from the satellite by sequentially power cycling the subsystems. The maximum perturbation came from the transmitter, and it proved to be $4 \mathrm{nT}$ at the boom-deployed magnetometer sensor (Merayo et al., 1998). In flight, when the field changes rapidly over the full scale range, the instrument's performance was limited by the 1-2 nT ADC-non-linearities. The Earth's field modelling residuals were less than $5 \mathrm{nT}_{\text {rms }}$, comparing favorably to the Ørsted modelling residuals of $2-3 \mathrm{nT}_{\mathrm{rms}}$, stemming mainly from the non-modelled magnetospheric perturbations (Merayo et al., 2002). Technical information on the instrument's noise and stability performance can be found in this paper and in references therein.

The electric field measurements are more difficult to calibrate since they are strongly dependent on the local plasma environment. However, for the quasi-static field and for a spacecraft in a low Earth orbit, the dominant component of the measured field is the field induced by the spacecraft motion. The accuracy is, therefore, primarily determined by the accuracy with which this induced field can be subtracted, which, in turn, depends primarily on the accuracy of the attitude determination. With an attitude knowledge of $0.1^{\circ}$, the induced field is known to be within approximately $0.5 \mathrm{mV} / \mathrm{m}$. The uncertainty added by the electronics is smaller. A possible DC offset in the differential voltage between opposing probes can be calibrated away since the spacecraft is spinning. Thus, the quasi-static electric field is normally accu- rate to well within $1 \mathrm{mV} / \mathrm{m}$. The frequency response of the analogue electronics depends on the plasma impedance. For example, with a plasma impedance of $100 \mathrm{M} \Omega$ in parallel with $2.2 \mathrm{pF}$, the $3 \mathrm{~dB}$ cutoff is at $6 \mathrm{kHz}$. With decreasing plasma impedance the cutoff frequency increases and vice versa. The frequency response is well within $3 \mathrm{~dB}$, up to the maximum sampling frequency of 2048 samples/s for most expected plasma impedances.

The ASC camera was also operating during the magnetometer calibration, and whenever the star sky was within the camera FOV, the boresight direction and the rotation about the boresight were determined in celestial coordinates. The intercalibration resulted in an angular pointing accuracy of 6 arc s between the output of the magnetometer sensor unit and the local magnetic field (Merayo, 1999; Merayo et al., 1998).

The EMMA data memory consists of $12 \mathrm{MB}$ of RAM for temporary storage of the measured samples and is needed for data recording when the satellite is not in contact with either of the ground stations or when the highest sampling rate is used. The memory is operated as a FIFO, which makes it a transparent telemetry queue that grows, shrinks, or is idle, depending on the sampling rate and on whether the telemetry stream is switched on or not. Part of the memory is used to hold LINDA data. Depending on the operational mode of LINDA, some fraction of the memory may be allocated as a buffer for LINDA data compression. Also stored in memory are data from the attitude sensors, as well as selected house- 

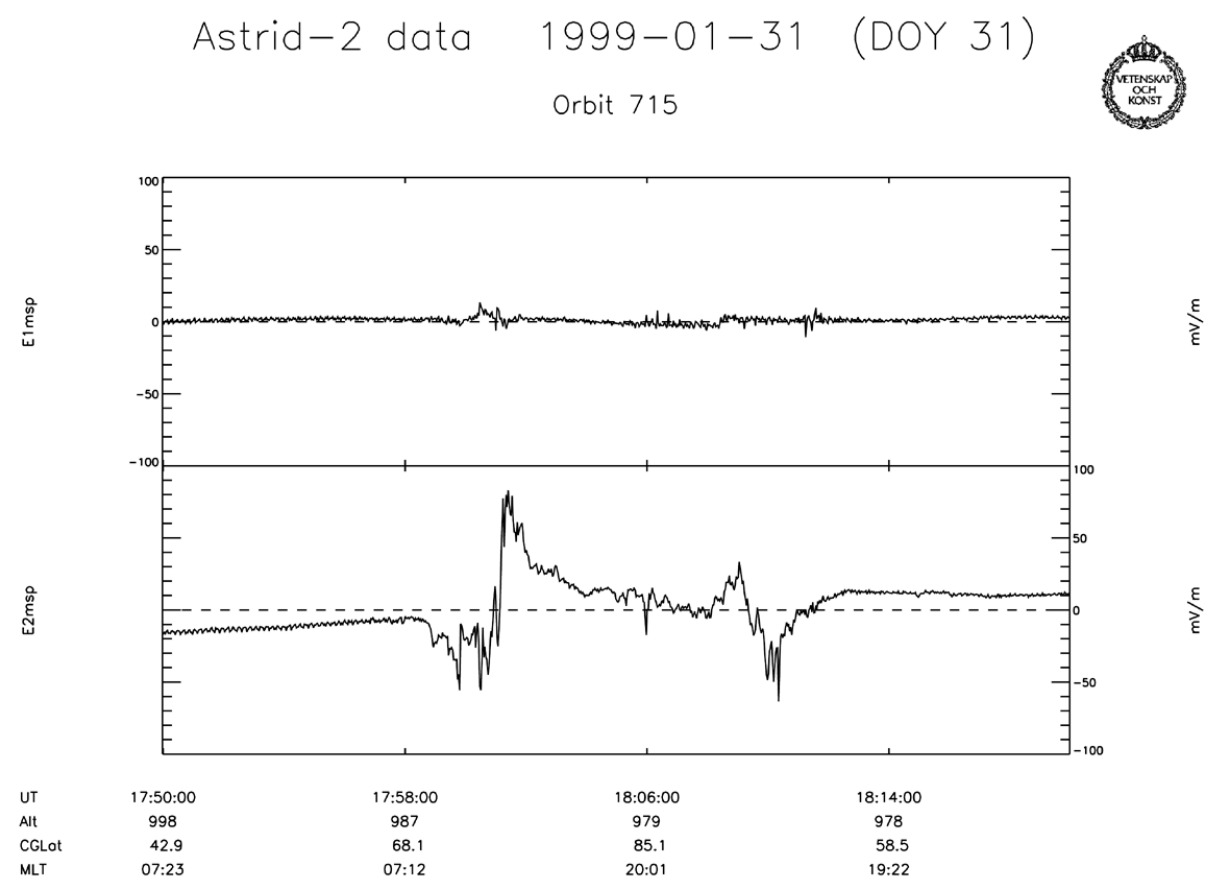

Fig. 5. Electric field data from a Northern Hemisphere polar pass showing the normal two-cell convection pattern with a dawn-to-dusk directed polar cap electric field.

Table 2. Summary of typical EMMA data memory capacity

\begin{tabular}{llll}
\hline Sampling rate & $16 \mathrm{~s}^{-1}$ & $256 \mathrm{~s}^{-1}$ & $2048 \mathrm{~s}^{-1}$ \\
Memory capacity & $8 \mathrm{~h}$ & $30 \mathrm{~min}$ & $4 \mathrm{~min}$
\end{tabular}

keeping and status data from EMMA, LINDA, and the ASU (Astrid-2 System Unit).

A typical memory allocation is $8 \mathrm{MB}$ for stored EMMA data, $2 \mathrm{MB}$ for stored LINDA data, and a $2 \mathrm{MB}$ temporary data area for LINDA data used for on-board processing and data compression. Assuming $8 \mathrm{MB}$ for EMMA and that the housekeeping data rate has been set low, approximate numbers for the time periods for which data can be held in memory are given in Table 2.

\section{Operational modes and instrument performance}

EMMA can be operated in a variety of different modes. In electric field mode (high-impedance mode), the sensors are biased with a known current (which may be zero, also known as keeping the sensor floating) and their electric potentials with respect to the spacecraft body are measured. By taking the difference in potential between opposing probes and dividing by their separation distance, the component of the electric field along the line-of-sight between the sensors is obtained. The potentials with respect to the satellite body of the four electric sensors are sampled in synchronism with the three orthogonal flux-gate sensors at either 16, 256, or
2048 samples per second. All four potentials are telemetred to ground which makes possible some interferometry, as well as estimation of the satellite potential by taking the average of the potentials of two or more probes. Another mode, mainly used for diagnostics, since it overlaps with the purpose of the LINDA instrument, is one where one of the four probes is operated in density mode, biased with a fixed voltage and the current running to it being measured. Since this is an auxiliary mode, the existing ADC (analogue-to-digital converter) normally used for housekeeping data is used for the current sampling rather than adding extra hardware, and thus, no housekeeping data sampling is performed when the instrument is running one probe in density mode.

Current sweeps may be performed in a highly flexible way. Sweeping is done by stepping either the current or the voltage through a sequence of known values while measuring the other quantity. The result of a sweep is a currentvoltage characteristic for the probe-plasma coupling, which may provide information on plasma density, plasma temperature, photoelectron temperature (if the sensor is sunlit), etc. Parameter tables may be uploaded which govern the current level stepping, and both the duration of the sweeps and their repetition frequency are individually adjustable. The sweeping may rotate between the probes or the same probe may be swept at each instance. Since the probe-plasma coupling is capacitive there is a minimum duration per current level for the measurement to make sense. Also, it is desirable that a sweep can be completed within a fraction of a satellite spin. Sweeping is normally not performed at the lowest sampling rate $\left(16 \mathrm{~s}^{-1}\right)$. 


$$
\begin{gathered}
\text { Astrid-2 data } 1999-02-11 \text { (DOY 42) } \\
\text { Orbit } 867
\end{gathered}
$$
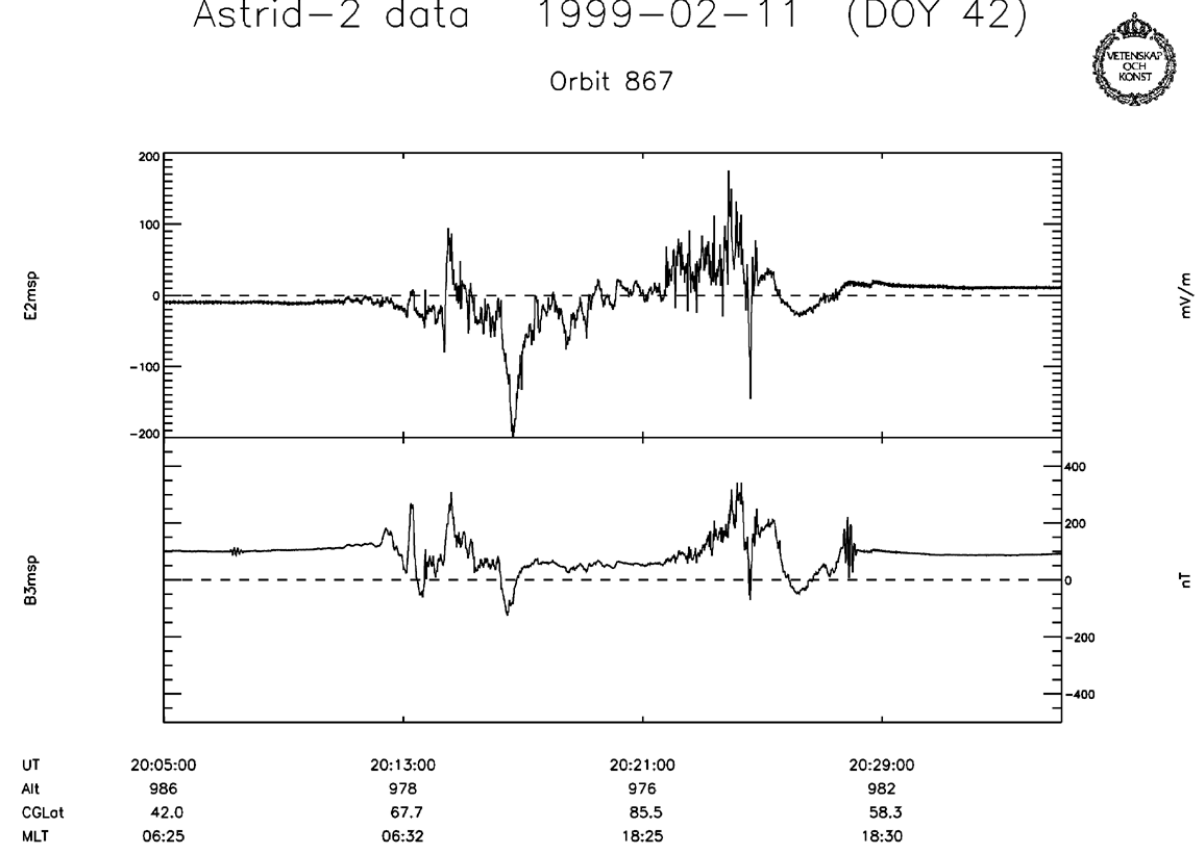

Fig. 6. Electric and magnetic field data from a polar pass through a transpolar auroral arc.

The rate of telemetring housekeeping data can be adjusted in binary steps from $1 / 4 \mathrm{~s}$ upward. Normally, the housekeeping data rate is selected so that the amount telemetred is some reasonably low fraction of the amount of science data. The time needed to dump the data memory to the ground is roughly 14 minutes, assuming there is $10 \mathrm{MB}$ 's worth of stored data and neglecting possible new data stored in memory while dumping. This is close to the length of a long ground station passage.

Figure 5 shows despun electric field data from a polar pass. E1msp and E2msp are both spin plane components. E1msp is along the projection of the magnetic field onto the plane and $\mathrm{E} 2$ is perpendicular to $\mathrm{B}$, positive in the duskward direction. The electric field induced by the spacecraft motion has been subtracted, so the plotted components refer to an inertial frame of reference. E1msp is seen to be small, which is to be expected for the quasi-parallel electric field at $1000 \mathrm{~km}$ altitude. E2msp shows auroral electric fields on either side of the polar cap and a duskward electric across the polar cap, consistent with a normal auroral oval and a two-cell convection pattern.

Figure 6 shows the duskward electric field (E2msp) and the sunward residual (IGRF subtracted) magnetic field (B3msp) for a polar pass through a transpolar auroral arc. Gradients in the sunward magnetic field indicate fieldaligned current sheets. The region 1 and region 2 current systems are clearly seen, as well as a current system associated with the transpolar arc. Current densities of the order of a few $\mu \mathrm{A} / \mathrm{m}^{2}$ are inferred. Co-located with the arc, and connected to the local current system, we also see sunward plasma convection. These data were obtained during a fairly unique transpolar arc event lasting at least five hours, during which Astrid-2 recorded signatures of several different field-aligned currents and associated plasma flow configurations in both hemispheres. More details are presented by Blomberg and Cumnock (submitted manuscript, Adv. Space Res., 2002).

\section{Summary}

EMMA is the electric and magnetic field instrument for the Astrid-2 micro-satellite. It measures two components of the electric field and three components of the magnetic field at sampling rates up to $2048 \mathrm{~s}^{-1}$. Novel technology was developed in order to fit the instrument into the tight microsatellite envelope. Most notably, a new wire boom system was flown, significantly reducing the mass compared to conventional systems, and the magnetometer was a highly digitalized flux-gate instrument.

EMMA, as well as other instruments on Astrid-2, have already provided material for several studies of the processes associated with the aurora, as evidenced by a number of papers that have already appeared in the literature, as well as several studies underway.

In conclusion, Astrid-2 and EMMA have demonstrated the feasibility of double-probe electric field measurements on micro-satellites. In addition, lessons on how to make future micro-satellite missions even more successful have been learned. Ample data for further study exist.

Acknowledgements. Topical Editor T. Pulkkinen thanks two referees for their help in evaluating this paper. 


\section{References}

Bak, T.: Spacecraft Attitude Determination - A Magnetometer Approach, Ph.D. Thesis, Ålborg University, Denmark, August 1999.

Blomberg, L. G., Lindqvist, P.-A., and Marklund, G. T.: Viking Observations of Electric Fields, in: Proc. of Symp. "Study of the Solar-Terrestrial System," Killarney, 16-19 June 1992, ESA SP-346, 269, 1992.

Blomberg, L. G., Marklund, G. T., Lindqvist, P.-A., and Bylander, L.: Astrid-2: An Advanced Auroral Microprobe, "Microsatellites as Research Tools," COSPAR Colloquia Series Vol. 10, Elsevier, 1999, 10, 57, 1999.

Blomberg, L. G., Marklund, G. T., Lindqvist, P.-A., Primdahl, F., Brauer, P., Bylander, L., Cumnock, J. A., Eriksson, S., Ivchenko, N., Karlsson, T., Kullen, A., Merayo, J. M. G., Pedersen, E. B., and Petersen, J. R.: The EMMA instrument on the Astrid2 micro-satellite, TRITA-ALP-2003-01, 2003.

Brauer, P, Risbo, T., Merayo, J. M. G., and Nielsen, O. V.: Fluxgate Sensor for the Vector Magnetometer Onboard the Astrid-2 Satellite, Sensors and Actuators, A Physical, 81, 184, 2000.

Eriksson, S., Blomberg, L. G., Ivchenko, N., Karlsson, T., and Marklund, G. T.: Magnetospheric response to the solar wind as indicated by the cross-polar potential drop and the low-latitude asymmetric disturbance field, Ann. Geophys., 19, 649, 2001.

Eriksson, S., Blomberg, L. G., and Weimer, D. R.: Comparing a Spherical Harmonic Model of the Global Electric Field Distribution With Astrid-2 Observations, J. Geophys. Res., 10.1029/2002JA009313, 2002.

Figueiredo, S.: Investigation of Subauroral Electric Fields in the Earth's Ionosphere Based on Astrid-2 Data, Royal Institute of Technology, Division of Plasma Physics Internal Report, ALP2001-101, 2001.

Hellman, H.: Design of Wire Boom System for a Satellite, Royal Institute of Technology, Division of Plasma Physics Internal Report, ALP-1996-101, 1996.

Holback, B., Jacksen, A., Åhlén, L., Jansson, S.-E., Eriksson, A. I., Wahlund, J.-E., Carozzi, T., and Bergman, J.: LINDA the Astrid-2 Langmuir probe instrument, Ann. Geophys., 19, 601, 2001.

Høymork, S. H., Yamauchi, M., Ebihara, Y., Narita, Y., Norberg, O., and Winningham, J. D.: Dense ion clouds of $0.1-2 \mathrm{keV}$ ions inside the CPS-region observed by Astrid-2, Ann. Geophys., 19, 621,2001

Ivchenko, N. and Marklund, G.: Observation of Alfvénic activity at $1000 \mathrm{~km}$ altitude, Ann. Geophys., 19, 643, 2001.

Ivchenko, N. and Marklund, G.: "Current Singularities" Observed on Astrid-2, Adv. Space Res., 30, 1779, 2002.

Ivchenko, N., Facciolo, L., Lindqvist, P.-A., Kekkonen, P., and Holback, B.: Disturbance of plasma environment in the vicinity of the Astrid-2 microsatellite, Ann. Geophys., 19, 655, 2001.

Jørgensen, J. L.: In orbit performance of a fully autonomous star tracker, ESA SP-425, 103, ESTEC, Noordwijk, The Netherlands, 2000.
Jørgensen, J. L., Denver, T., Betto, M., and Van den Braembussche, P.: The PROBA star tracker performance, in: Small satellites for Earth observation, edited by Röser, H. P., Sandau, R., and Valenzuela, A., 201, Wissenschaft und Technik Verlag, Berlin, 2003.

Karlsson, T., Marklund, G. T., Blomberg, L. G., and Mälkki, A.: Subauroral Electric Fields Observed by the Freja Satellite, a Statistical Study, J. Geophys. Res., 103, 4327, 1998.

Keith, W. R., Winningham, J. D., and Norberg, O.: A new, unique signature of true cusp, Ann. Geophys., 19, 611, 2001.

Kurth, W. S., Hospodarsky, G. R., Gurnett, D. A., Kaiser, M. L., Wahlund, J.-E., Roux, A., Canu, P., Zarka, P., and Tokarev, Y.: An overview of observations by the Cassini radio and plasma wave investigation at Earth, J. Geophys. Res., 106, 30 239, 2001.

Marklund, G. T., Blomberg, L. G., Fälthammar, C.-G., and Lindqvist, P.-A.: On Intense Diverging Electric Fields Associated With Black Aurora, Geophys. Res. Lett., 21, 1859, 1994.

Marklund, G., Karlsson, T., and Clemmons, J.: On Low-Altitude Particle Acceleration and Intense Electric Fields and their Relationship to Black Aurora, J. Geophys. Res., 102, 17 509, 1997a.

Marklund, G., Blomberg, L., Bylander, L., and Lindqvist, P.-A.: Astrid-2, A Low-Budget Microsatellite Mission for Auroral Research, in: Proc. of 13th ESA Symposium on European Rocket and Balloon Programmes and Related Research, Öland, Sweden, 26-29 May 1997, ESA SP-397, 387, 1997b.

Marklund, G. T., Blomberg, L. G., and Persson, S.: Astrid-2, an advanced microsatellite for auroral research, Ann. Geophys., 19, 589, 2001a.

Marklund, G., Karlsson, T., Eglitis, P., and Opgenoorth, H.: Astrid2 and ground-based observations of the auroral bulge in the middle of the nightside convection throat, Ann. Geophys., 19, 633, 2001b.

Merayo, J. M. G.: Magnetic Gradiometry, Ph.D. Thesis, Institute of Automation, Technical University of Denmark, 1999.

Merayo, J. M. G., Brauer, P., Risbo, T., Pedersen, E. B., Petersen, J. R., and Primdahl, F.: Astrid-2 EMMA Magnetic Calibration, Final Report, Institute of Automation, Technical University of Denmark, Lyngby, Denmark, 1998.

Merayo, J. M. G., Brauer, P., Primdahl, F., Jørgensen, P. S., Risbo, T., and Cain, J.: The spinning Astrid-2 satellite used for modeling the Earth's magnetic field, IEEE Trans. Geoscience Electronics and Remote Sensing, 40, 898, 2002.

Norberg, O., Winningham, J. D., Lauche, H., Keith, W., Puccio, W., Olsen, J., Lundin, K., and Scherrer, J.: The MEDUSA electronand ion spectrometer and the ultraviolet PIA photometers on Astrid-2, Ann. Geophys., 19, 593, 2001.

Pedersen, E. B., Primdahl, F., Petersen, J. R., Merayo, J. M. G., Brauer, P., and Nielsen, O. V.: Digital fluxgate magnetometer for the Astrid-2 satellite, Meas. Sci. Technol., 10, N124, 1999.

Piil-Henriksen, J., Merayo, J. M. G., Nielsen, O. V., Petersen, H., Raagaard Petersen, J,. and Primdahl, F.: Digital detection and feedback fluxgate magnetometer, Meas. Sci. Technol., 7, 1, 1996. 